\title{
The rise of a ribosomopathy and increased cancer risk
}

Friedrich Luft

Accepted: 26 November 2009 / Published online: 12 December 2009

(C) Springer-Verlag 2009

Diamond-Blackfan anemia (DBA), a rare congenital disorder characterized by a decrease in erythroid precursor cells, has recently become a model for genetic diseases linked to mutations in genes encoding ribosomal proteins or factors involved in ribosomal biogenesis [1]. Such diseases should be termed ribosomopathies. The clinical relevance is twofold. First, they may involve the cell nucleolus, which is not a common clinical backyard. Second, the ribosomopathies draw attention to the ribosome and its entire endoplasmatic reticulum, functions, structures, protein fates, cell death, and survival. These topics are indeed highly clinically relevant. DBA is characterized by anemia (low red blood cell counts) with decreased erythroid progenitors in the bone marrow [2]. The condition usually develops during the neonatal period. About half of affected individuals also have a variety of congenital abnormalities, including craniofacial malformations, thumb or upper limb abnormalities, cardiac defects, urogenital malformations, and cleft palate. Low birth weight and generalized growth delay are also sometimes observed. DBA is genetically and clinically heterogeneous. DBA patients have a modest risk of developing leukemias, the myelodysplastic syndromes (MDS), and other cancers. Cells from DBA patients display anomalies in the maturation of ribosomal RNA. The explanation of this unexpected link between ribosome biogenesis, a ubiquitous process, and a disease mostly affecting erythroid differentiation may stem in part from the emerging concept of the "ribosomal stress response", a signaling pathway triggering cell cycle arrest in response to a defect in ribosome synthesis.

F. Luft $(\bowtie)$

Experimental and Clinical Research Center, Franz-Volhard Clinic, Berlin, Germany

e-mail: luft@charite.de
The inheritance of DBA can be either autosomaldominant or recessive [2]. About $25 \%$ of DBA patients harbor a mutation in the ribosomal protein S19 gene (RPS19); another $20 \%$ carry mutations in other ribosomal protein genes. The mechanisms by which RPS19 mutations might eventually result in cancer development are unknown; however, additional mutations to attain clonal dominance are postulated. At least 77 RPS19 mutations have been described so far [3], most of which represent gene deletions, translocations, nonsense, or frame shift mutations. While the homozygous mouse Rps19 gene deletion was lethal and the heterozygous one did not express an obvious phenotype [2], Rps19 gene knockdowns in Zebra fish produced a phenotype resembling DBA. Furthermore, the novel mouse Rps $19^{\text {Dsk }}$ recapitulated the human DBA phenotype fairly well in terms of anemia with growth retardation. McGowan et al. reported on two mouse dark-skinned (Dsk) loci caused by mutations in Rps19 and Rps20 (ribosomal protein S20) [4]. They pointed out that mutations in genes encoding ribosomal proteins cause the Minute phenotype in Drosophila and mice, and DBA in humans. They identified a common pathophysiologic program in which p53 stabilization stimulated Kit ligand expression. Moreover, epidermal melanocytosis was stimulated via a paracrine mechanism. Kit (also CD117 or stem cell factor-receptor) is a cell membrane-expressed tyrosine kinase receptor. The authors observed that accumulation of p53 caused reduced body size and lowered erythrocyte count in their $\operatorname{Rps} 19^{\text {Dsk }}$ mice. These results provide a mechanistic explanation for the diverse collection of phenotypes that accompany reduced dosage of genes encoding ribosomal proteins, and have implications for understanding normal human variation and human disease (Fig. 1). The authors suggested that their findings make sense, since DBA features cells with a pro-apoptotic 


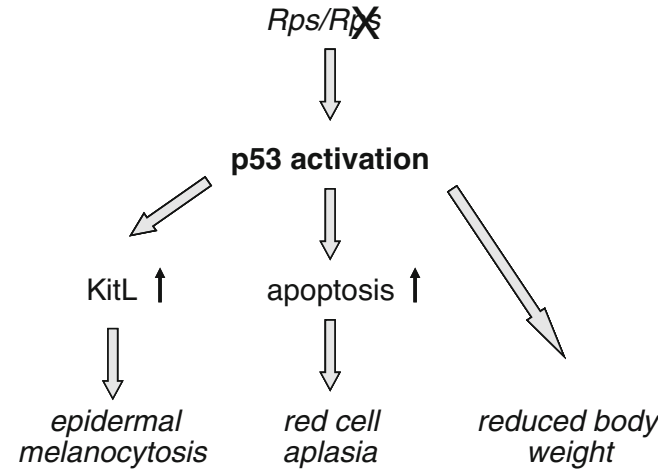

Fig. 1 Pleiotropic phenotype of Rps $19^{\text {Dsk }}$ mouse resembling human DBA. Reduced dosage of ribosomal protein $\mathrm{S}$ genes $(R p s)$ induces $\mathrm{p} 53$ activation/stabilization resulting in KitL-dependent epidermal malonocytosis, red cell aplasia, and reduced body weight (adapted from [4])

phenotype. As a result, p53 stabilization and activation could be important features of the disease. In fish and mouse models, p53 mutations could therefore "rescue" the phenotype. The findings reported by McGowan et al. support the notion that reduced dosage of genes encoding ribosomal proteins could contribute to the disease [4].

The nucleolus (Fig. 2) is a non-membrane bound structure composed of proteins and nucleic acids found within the cell nucleus. Ribosomal RNA is transcribed and assembled within the nucleolus. Thus, the genesis of ribosomopathy largely takes place in the nucleolus. Evidence suggests several potential mechanisms whereby abortive ribosome assembly or nucleolar stress could signal p53 activation. Certain ribosomal proteins interact with murine double minute-2 (MDM2), a RING finger ubiquitin ligase that promotes the degradation of $\mathrm{p} 53$. Other ribosomal proteins bind to MDM2, reduce its ubiquitin ligase activity, which stabilizes $\mathrm{p} 53$. Thus, ribosomal proteins that are diverted from their normal fates or altered through mutation could be important signaling molecules linking abortive ribosomal assembly to $\mathrm{p} 53$ activation and stabilization.

Why DBA patients, as well as others suffering from bone marrow failure syndromes, should be prone to develop malignancies such as leukemia and MDS is unclear. Presumably, additional mutations must occur in DBA patients to reach clonal dominance. In this issue, Fröjmark et al. [5] investigated a possible interaction that could lead to malignancy. The proto-oncogene serine/threonine-protein kinase (PIM-1) is an enzyme that in humans is encoded by the PIM1 gene. The PIM-1 kinase is believed to have diverse biological roles in cell survival, proliferation, and differentiation. In humans, PIM-1 is often expressed in both normal and transformed cells. The PIM-1 kinase is a true oncogene that is implicated in early transformation and tumor progression in hematopoietic malignancies and prostate cancer [6]. PIM-1 could possibly be a promising target for drug development programs. However, the complexity of its molecular structure has posed challenges in the development of PIM-1 inhibitors. Fröjmark et al. reasoned that RPS19 and PIM-1 mutations could contribute to malignancy in DBA patients. The authors report that PIM-1 is ubiquitously expressed in hematopoietic tissue, where it is involved in the regulation of cell proliferation and survival. The genedeleted mouse has anemia and impaired erythropoiesis. PIM1 interacts with c-Myc, another proto-oncogene that is important to cell growth control, differentiation, and apopto-

Fig. 2 Diamond-Blackfan anemia is a genetic erythrocyte precursor failure syndrome causing severe anemia in newborns. Later-on cancer commonly develops. Ribosomal protein haplo-insufficency (RPS19 mutations) results in the accumulation of intermediates that interfere with nucleolar function. Malignancy is largely a function of Myc and p53 pathways. Fröjmark et al. [5] showed that absence of the tyrosine kinase, PIM-1 favors the development of malignancy in this "ribosomopathy" (adapted from [2])

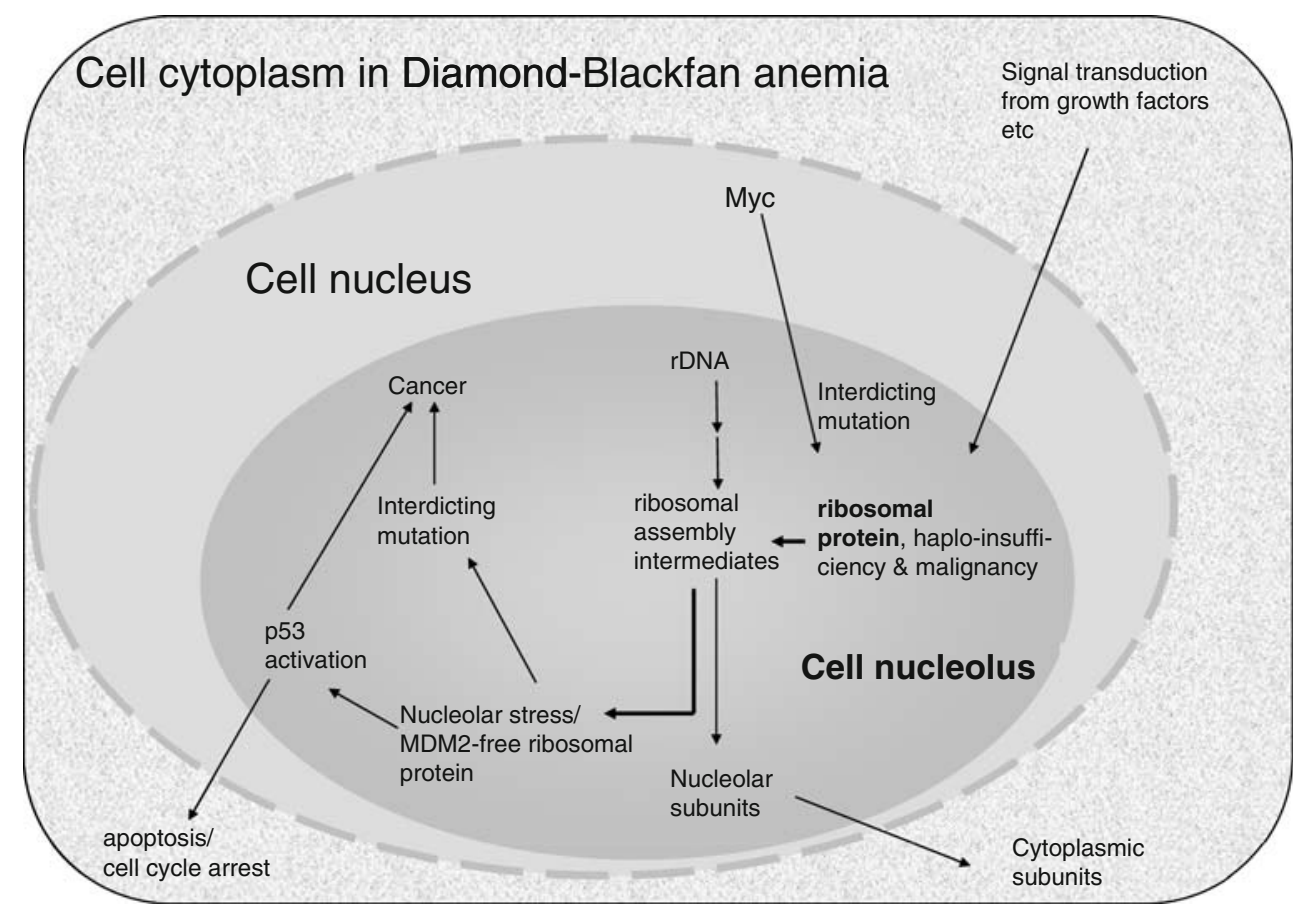


sis. In contrast to patients bearing RPS19 mutations, the Rps $19^{+/}$mouse does not express an obvious phenotype. Thus, the authors crossed RPS ${ }^{+/}$mice with Pim- $1^{-/-}$mice to test the notion that these signaling pathways interact with one another.

The authors [5] produced a double-mutant mouse (Rps$19^{+/-} \mathrm{Pim}_{-1^{-/}}$) and corresponding controls (all permutations and combinations) and as a result, the double-mutant mice

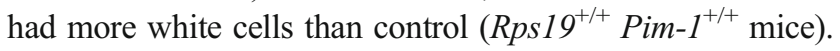
Whereas c-Myc and the anti-apoptotic factors, Bcl2, BclXL, and Mcl-1 were upregulated, the apoptotic factors Bak and Caspase 3, as well as p21 were downregulated. The authors argue that their findings suggest an Rps19 and Pim-1 deficiency that promotes increased myeloid cell growth through a deregulation of c-Myc and upregulation of antiapoptotic Bcl proteins. How can we put these pieces together? The proto-oncogene Myc (c-Myc) encodes a transcription factor that regulates the transcription of many different genes. When c-Myc is mutated or overexpressed, the protein does not bind correctly, and could cause cancer (or perhaps MDS). c-Myc binds to enhancer box sequences (E-boxes) and recruits histone acetyltransferases.

$\mathrm{Bcl} 2$ is another human proto-oncogene; its product is an integral membrane protein located in the membranes of the endoplasmic reticulum, nuclear envelope, and in the outer membranes of the mitochondria. Miyake et al. [7] analyzed the effects of RPS19 deficiency on erythropoietin-induced signal transduction, cell cycle, and apoptosis in an RPS19deficient human erythroleukemic cell line, TF-1 cells. They found that RPS19-deficient TF-1 cells showed G0/G1 arrest together with accumulation of p21 and p27. Western blot analysis of apoptosis-related proteins in TF-1 cells showed that while the levels of $\mathrm{Bcl} 2$ and Bad were decreased that of Bax was increased. Their findings indicate that RPS19 deficiency causes apoptosis and accelerated loss of erythroid progenitors in RPS19-deficient DBA cells. Interestingly, Fröjmark and colleagues found that when Pim-1 deficiency is added to $\mathrm{Rps1} 19^{+-}$in vivo, Bcl2 was upregulated [5]. Since c-Myc acts as a molecular switch of the Bcl2 family, the balance of pro-apoptotic and anti-apoptotic Bcl family members could result in reduced apoptosis. Thus, the pathways identified by the authors could have important implications for DBA and could perhaps shed light on the genesis of MDS. The latter is an everyday occurrence on the wards and much more common than DBA.

Myc activation could compensate for a ribosomal protein deficiency allowing for the emergence of clones with a survival advantage predisposing to malignancy. Because numerous signaling pathways involved in growth and cell division regulate ribosomal synthesis, numerous other targets could also exist to explain the increased cancer incidence in DBA patients [8]. As a matter of fact, heterozygous mutations in various ribosomal protein genes were recently described in Zebra fish that resulted in peripheral nerve sheath tumors [9]. A schematic view modified from Lipton and Ellis [2] suggests that ribosomal protein genes could act as tumor suppressors (Fig. 2). Excitement here evolves from cancer schemes derived from obscure clinical syndromes that could give us leverage to much more common diseases in the general population.

What are the clinical implications of the double (one heterozygous) knockout model to DBA and subsequent malignancy if any? The pathways identified by the authors, namely upregulated c-Myc, Bcl2, and Mcl-1 are interesting. Missing here are clinical data that would require an analysis of bone marrow from DBA patients. Is PIM-1 altered in the cells of DBA patients developing MDS or leukemia? Could a tyrosine kinase inhibitor be of clinical utility? Does the PIM-1 pathway suggest other avenues that could be translated to MDS patients in general? After all, MDS is a common malignant condition in our ever-aging society. The authors provided a model emanating from an obscure disease involving ribosomal proteins that clinicians do not encounter every day. Plenty of thought-food is left for future research.

Respectfully,

Friedrich C. Luft

\section{References}

1. Aguissa-Touré AH, Da Costa L, Leblanc T, Tchernia G, Fribourg S, Gleizes PE (2009) Diamond-Blackfan anemia reveals the dark side of ribosome biogenesis. Med Sci (Paris) 25:69-76

2. Lipton JM, Ellis SR (2009) Diamond-Blackfan anemia: diagnosis, treatment, and molecular pathogenesis. Hematol Oncol Clin North Am 23:261-282

3. Campagnoli MF, Ramenghi U, Armiraglio M, Quarello P, Garelli E, Carando A, Avondo F, Pavesi E, Fribourg S, Gleizes PE, Loreni F, Dianzani I (2008) RPS19 mutations in patients with DiamondBlackfan anemia. Hum Mutat 29:911-920

4. McGowan KA, Li JZ, Park CY, Beaudry V, Tabor HK, Sabnis AJ, Zhang W, Fuchs H, de Angelis MH, Myers RM, Attardi LD, Barsh GS (2008) Ribosomal mutations cause p53-mediated dark skin and pleiotropic effects. Nat Genet 40:963-970

5. Fröjmark AS, Badhai J, Klar J, Thuveson M, Schuster J, Dahl N (2010) Cooperative effect of ribosomal protein s19 and Pim-1 kinase on murine c-Myc expression and myeloid/erythroid cellularity. J Mol Med. doi:10.1007/s00109-009-0558-9

6. Shah N, Pang B, Yeoh KG, Thorn S, Chen CS, Lilly MB, Salto-Tellez M (2008) Potential roles for the PIM1 kinase in human cancer-a molecular and therapeutic appraisal. Eur J Cancer 44:2144-2151

7. Miyake K, Utsugisawa T, Flygare J, Kiefer T, Hamaguchi I, Richter J, Karlsson S (2008) Ribosomal protein S19 deficiency leads to reduced proliferation and increased apoptosis but does not affect terminal erythroid differentiation in a cell line model of DiamondBlackfan anemia. Stem Cells 26:323-329

8. Ruggero D, Pandolfi PP (2003) Does the ribosome translate cancer? Nat Rev Cancer 3:179-192

9. Amsterdam A, Sadler KC, Lai K, Farrington S, Bronson RT, Lees JA, Hopkins N (2004) Many ribosomal protein genes are cancer genes in zebrafish. PLoS Biol 2:E139 